\title{
Onset of Patterns in an Ocillated Granular Layer: Continuum and Molecular Dynamics Simulations
}

\author{
J. Bougie, J. Kreft, J. B. Swift, and Harry L. Swinney \\ Center for Nonlinear Dynamics and Department of Physics, \\ University of Texas at Austin, Austin, TX 78712
}

(Dated: June 19, 2018)

\begin{abstract}
We study the onset of patterns in vertically oscillated layers of frictionless dissipative particles. Using both numerical solutions of continuum equations to Navier-Stokes order and molecular dynamics (MD) simulations, we find that standing waves form stripe patterns above a critical acceleration of the cell. Changing the frequency of oscillation of the cell changes the wavelength of the resulting pattern; MD and continuum simulations both yield wavelengths in accord with previous experimental results. The value of the critical acceleration for ordered standing waves is approximately $10 \%$ higher in molecular dynamics simulations than in the continuum simulations, and the amplitude of the waves differs significantly between the models. The delay in the onset of order in molecular dynamics simulations and the amplitude of noise below this onset are consistent with the presence of fluctuations which are absent in the continuum theory. The strength of the noise obtained by fit to Swift-Hohenberg theory is orders of magnitude larger than the thermal noise in fluid convection experiments, and is comparable to the noise found in experiments with oscillated granular layers and in recent fluid experiments on fluids near the critical point. Good agreement is found between the mean field value of onset from the Swift-Hohenberg fit and the onset in continuum simulations. Patterns are compared in cells oscillated at two different frequencies in MD; the layer with larger wavelength patterns has less noise than the layer with smaller wavelength patterns.
\end{abstract}

PACS numbers: 45.70.Qj,05.40.Ca,47.54.+r

\section{INTRODUCTION}

\section{A. Background}

A successful hydrodynamic theory of granular media could allow scientists and engineers to exploit the powerful techniques of fluid dynamics to describe granular phenomena. Recent experiments [1, 2] and simulations [3] demonstrate the potential for hydrodynamic theory to describe granular media; however, the validity of such methods has not yet been established for a general description of granular flow phenomena [4, 5, 6].

Several proposed rapid granular flow models use equations of motion for continuum fields - number density $n$, velocity $\mathbf{u}$, and granular temperature $T\left(\frac{3}{2} T\right.$ is the average kinetic energy due to random particle motion) [7, 8, 9] . In one approach, particle interactions are modeled with binary, inelastic hard-sphere collision operators in kinetic theory to derive continuum equations to Euler [10], Navier-Stokes [11], and Burnett [12] order. In this paper, we use 3D simulations of continuum equations to Navier-Stokes order and 3D inelastic hard-sphere molecular dynamics (MD) simulations to investigate the onset of standing wave patterns in vertically oscillated granular layers.

\section{B. Standing wave patterns in oscillated granular layers}

Vertically oscillated layers have provided an important testbed for granular research. Flat layers of grains on a plate oscillating sinusoidally in the direction of gravity exhibit convection [13], clustering [14], shocks [15], steady-state flow fields far from the plate [16], and standing wave pattern formation [17].

A layer of grains on a plate oscillating sinusoidally in the direction of gravity with frequency $f$ and amplitude $A$ will leave the plate at some time in the cycle if the maximum acceleration of the plate is greater than that of gravity. The layer dilates above the plate, then collides with the plate later in the cycle and is compressed on the plate by this collision. Above a critical value of acceleration, standing wave patterns spontaneously form in the layer. This pattern is subharmonic with respect to the plate, repeating every $2 / f[17]$.

Various subharmonic standing wave patterns, including stripe, square, and hexagonal patterns, have been found experimentally, depending on the nondimensional frequency $f^{*}=f \sqrt{H / g}$ and the nondimensional accelerational amplitude $\Gamma=A(2 \pi f)^{2} / g$, where $H$ is the depth of the layer as poured, and $g$ is the acceleration due to gravity [17].

Studies using hydrodynamic equations have not yet yielded the standing wave patterns observed in experiments. Here we investigate the onset of ordered standing wave patterns using fully three-dimensional (3D) simulations of continuum equations to Navier-Stokes order as well as molecular dynamics (MD) simulations. We use a continuum model for frictionless, inelastic particles, and investigate the onset of stripe patterns. 


\section{Fluctuating hydrodynamics}

Near the onset of convection patterns in RayleighBénard convection of fluids, fluctuations caused by thermal noise create deviations from dynamics predicted from linear theory. These fluctuations are described by the addition of terms to the Navier-Stokes equations; this theory is known as fluctuating hydrodynamics [18, 19, 20]. Recent experiments have shown that fluctuating hydrodynamics theory accurately describes the dynamics of fluids near the onset of convection [21, 22, 23].

Experimental investigations of coherent fluctuations and pattern formation in oscillated granular layers have indicated that fluctuations due to the movement of individual grains play a much more significant role in the collective behavior of granular media than do thermal fluctuations in ordinary fluids 24]. Thus, a consistent theory of granular hydrodynamics may need to include fluctuations.

\section{Model system}

We simulate a layer of grains on an impenetrable plate which oscillates sinusoidally in the direction of gravity. The layer depth at rest is approximately $H=5.4 \sigma$, where the grains are modeled as identical, frictionless spheres with diameter $\sigma$ and coefficient of restitution $e=0.7$. For most of the paper, we study the onset of patterns as a function of $\Gamma$, while the frequency of plate oscillation is held constant at $f^{*}=0.4174$. This corresponds to a frequency of $56 \mathrm{~Hz}$ for particles with a diameter of 0.1 mm. For $\Gamma \gtrsim 2.5$, stripes are seen experimentally for a range of parameters, including $f^{*}=0.4174, H=5.4$ [17]. In Sec. IIIB and Sec. IVC, frequency is varied to investigate the effect of changing frequency on pattern formation.

Experiments [25] and MD simulations [26] indicate that inter-particle friction plays an important role in the standing wave patterns. MD simulations with friction between particles have quantitatively reproduced the stripe, square, and hexagonal subharmonic standing wave patterns seen experimentally for a wide range of parameters [27]. However, MD simulations using frictionless particles do not yield stable square or hexagonal patterns, but only yield stripe patterns, and exhibit the onset of patterns at lower $\Gamma$ than that seen for frictional particles [26]. This result is consistent with experiments which show that reducing friction by adding graphite can destabilize square patterns [25]. In this study, we neglect the effects of friction in our continuum and MD simulations, and study only the onset of stripe patterns in frictionless layers. To investigate other patterns such as squares or hexagons, simulations would need to include friction between particles.

We use MD and continuum simulations to investigate the dynamics of this system near onset, and use simulations of the Swift-Hohenberg ( $\mathrm{SH}$ ) model equation to compare our results between the two. Section II describes the methods used to simulate and analyze these patterns, Sec. III compares patterns formed in MD and continuum simulations. Section IV compares MD simulations to Swift-Hohenberg theory, and Sec. V presents our conclusions.

\section{METHODS}

\section{A. Molecular dynamics simulation}

We use an inelastic hard sphere molecular dynamics simulation, which was previously used in conjunction with the continuum simulation used in this paper to model shock waves in a granular shaker [28]. This same MD code with friction added has been found to describe well the patterns observed in experiments on oscillating granular layers [27, 29].

The collision model assumes instantaneous binary collisions in which energy is dissipated, as characterized by the normal coefficient of restitution $e$. We neglect surface friction between particles, as well as between the particles and the plate. To prevent inelastic collapse, we use a coefficient of restitution which depends on the relative colliding velocity of the particles $v_{n}$ : $e\left(v_{n}\right)=$ $1-0.3\left(v_{n} / \sqrt{g \sigma}\right)^{3 / 4}$ for $v_{n}<\sqrt{g \sigma}$, and $e=0.7$ otherwise [27].

The MD simulations are calculated in a box of size $L_{x} \times L_{y}$ in the horizontal directions $x$ and $y$, where $L_{x}$ and $L_{y}$ are varied to investigate patterns with different wavelengths. The simulations use periodic boundary conditions in the horizontal directions, an impenetrable lower plate which oscillates sinusoidally between $z=0$ and $z=2 A$, and an upper plate fixed at a height $z=200 \sigma$, as in the previous investigation of shock propagation [28].

For each MD simulation, $\left(L_{x} / \sigma\right) \times\left(L_{y} / \sigma\right) \times 6$ particles were used. In actual packings seen experimentally, $6 / \sigma^{2}$ particles per unit area of the bottom plate corresponds to a layer depth $H=5.4 \sigma$ as poured, representing a volume fraction $\nu \approx 0.58$. [27]. The total mass of the layer matches that of the continuum simulations.

\section{B. Continuum simulation}

We use a continuum simulation previously used to model shock waves in a granular shaker [28]. Our simulation numerically integrates continuum equations of Navier-Stokes order proposed by Jenkins and Richman 11] for a dense gas composed of frictionless (smooth), inelastic hard spheres. We integrate these hydrodynamic equations to find number density, momentum, and granular temperature, using a second order finite difference scheme on a uniform grid in $3 \mathrm{D}$ with first order adaptive time stepping [28].

As in our MD simulations, the granular fluid in the continuum simulations is contained between two impen- 
etrable horizontal plates at the top and bottom of the container, where the lower plate oscillates sinusoidally between height $z=0$ and $z=2 A$. In our MD simulations, the ceiling is fixed in space at a height of $z=200 \sigma$, but to minimize computation time, the ceiling in continuum simulations is located at height $80 \sigma$ above the lower plate and oscillates with the bottom plate. In our previous study of shock formation, changing the ceiling height from $200 \sigma$ to $80 \sigma$ resulted in a fractional root mean square difference of less than $1 \%$ in the shock location over one cycle [28].

As in the MD simulations, we use periodic horizontal boundary conditions and boxes of size $L_{x} \times L_{y}$ in the horizontal directions $x$ and $y$, where $L_{x}$ and $L_{y}$ are varied. In each case, continuum simulations are compared to MD simulations with the same horizontal dimensions $L_{x}$ and $L_{y}$. The numerical methods, boundary conditions at the top and bottom plate, and grid spacing are the same as used in the previous study of shocks [28].

The energy loss due to collisions in continuum simulations is characterized by a single parameter, the normal coefficient of restitution $e=0.70$. Throughout this paper, we use units such that particles in MD simulations have mass unity, and the total mass of the layer in the continuum simulations matches that used in MD simulations.

\section{Characterizing patterns}

To visualize peaks and valleys formed by standing wave patterns, we calculate the height of the center of mass of the layer, $z_{c m}(x, y, t)$ as a function of horizontal location in the cell at various times in the cycle. At a given time $t_{0}$ and horizontal location $\left(x_{0}, y_{0}\right), z_{c m}\left(x_{0}, y_{0}, t_{0}\right)$ is the center of mass of all particles whose horizontal coordinates lie within a bin of size $\Delta x_{b i n} \times \Delta y_{b i n}$ centered at $\left(x_{0}, y_{0}\right)$. For continuum simulations, we use the simulation grid size to define the bins: $\Delta x_{b i n}=\Delta x=2 \sigma$ and $\Delta y_{b i n}=\Delta y=2 \sigma$. For MD simulations, we use bins of size $2 \sigma \times 2 \sigma$ in Section III to compare to continuum simulations with the same bin size. Peaks in the pattern correspond to maxima of $z_{c m}$, and valleys correspond to minima.

To measure the amplitude of patterns and fluctuations in continuum and MD simulations, we examine the deviation of the height of the center of mass of the layer as a function of horizontal location in the cell from the center of mass height averaged over the cell at that phase in the cycle:

$$
\psi(x, y, t)=z_{c m}(x, y, t)-\left\langle z_{c m}(x, y, t)\right\rangle,
$$

where $x$ and $y$ are the horizontal coordinates, $t$ is the time in the cycle, $z_{c m}(x, y)$ is the height of the center of mass of the layer at horizontal location $(x, y)$, and the brackets represent an average over all horizontal locations in the cell at a given time $t$.
Throughout this paper, we characterize the patterns at the beginning of a sinusoidal oscillation cycle, such that the plate is at its equilibrium position and moving upwards. Using this definition, $\left\langle\psi^{2}(t)\right\rangle$ represents the mean square deviation of the height of the layer from the mean height of the layer at that phase of the plate. We note that $\left\langle\psi^{2}\right\rangle$ is large for layers with high amplitude patterns or fluctuations, and goes to zero as the layer becomes perfectly flat.

In addition to $\left\langle\psi^{2}\right\rangle$, we distinguish between ordered patterns (stripes) and disordered fluctuations by characterizing the long range order of the pattern. To characterize the long range order of the patterns, we first calculate the power spectrum of the pattern: $S\left(k_{x}, k_{y}, t\right)=\left|\tilde{\psi}\left(k_{x}, k_{y}, t\right)\right|^{2}$, where $\tilde{\psi}\left(k_{x}, k_{y}, t\right)=$ $\int_{0}^{L_{x}} \int_{0}^{L_{y}} \psi(x, y, t) e^{-i k_{x} x} e^{-i k_{y} y} d x d y$. We then transform to polar coordinates in $k$ space: $k_{r}=\sqrt{k_{x}^{2}+k_{y}^{2}}, k_{\theta}=$ $\tan ^{-1}\left(k_{y} / k_{x}\right)$ to find $S\left(k_{r}, k_{\theta}\right)$ in the range $0 \leq k_{\theta}<\pi$. We integrate radially to find the angular orientation of the power spectrum: $S\left(k_{\theta}\right)=\int_{0}^{K} S\left(k_{r}, k_{\theta}\right) k_{r} d k_{r}$, where $K=\frac{2 \pi \Delta x_{b i n}}{L_{x}}$. We bin $k_{\theta}$ into 21 bins between $k_{\theta}=0$ and $k_{\theta}=\pi$, and characterize the long range order of the patterns by the fraction of the total integrated power that lies in the bin with the maximum power:

$$
P_{\max }=\frac{S_{\max }(\theta)}{\int_{0}^{\pi} S\left(\theta_{i}\right) d k_{\theta}},
$$

where $S_{\max }(\theta)$ is the integrated power within an angle $\pi / 21$ of the maximum value of $S(\theta)$. Thus $P_{\max }$ is the fraction of the total power that lies within approximately $\pi / 21$ of the angular location of the maximum power. For a perfectly disordered state, with equal power in all directions, $P_{\max }$ would approach $\frac{1}{21} \approx 0.05$, while $P_{\max }=1$ for a state with all power in a single bin. Thus $P_{\max }$ provides a measure of order when stripes form.

\section{PATTERN ONSET AND DISPERSION}

\section{A. Stripe patterns}

Experimental investigations of shaken granular layers have shown that above a critical acceleration of the plate $\Gamma_{c}$, standing wave patterns form spontaneously. These patterns oscillate subharmonically, repeating every $2 / f$, so that the location of a peak of the pattern becomes a valley after one cycle of the plate, and vice versa [17].

Continuum and MD simulations produce standing wave patterns for $\Gamma=2.2$ and $f^{*}=0.4174$ (Fig. (1). Alternating peaks and valleys form a stripe pattern which oscillates at $f / 2$ with respect to the plate oscillation; a location in the cell which represents a peak during one cycle will become a valley the next cycle, and then return to a peak on the following cycle. For a box of size $126 \sigma \times 126 \sigma$ in the horizontal direction, six wavelengths 


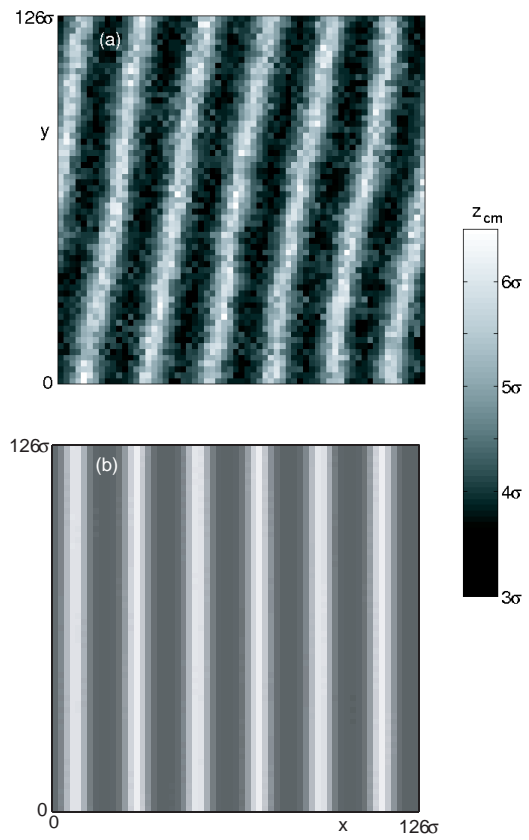

FIG. 1: An overhead view of a layer of grains, showing the center of mass height $z_{c m}$ as a function of horizontal position $(x, y)$ in a cell with horizontal dimensions $L_{x} \times L_{y}=$ $126 \sigma \times 126 \sigma$, from (a) MD simulations and (b) continuum simulations. Peaks of the layer corresponding to large center of mass height $z_{c m}$ are shown in white; valleys corresponding to low $z_{c m}$ are shown in black.

fit in the box in both MD and continuum simulations, yielding a wavelength of $21 \sigma \pm 4 \sigma$ in both continuum and MD simulations (Fig. 11) .

\section{B. Dispersion Relations in Continuum, MD, and Experiment}

Experiments have shown that the wavelength $\lambda$ of standing wave patterns in shaken granular layers depends on the frequency of the plate oscillation 30, 31, 32]. For a range of layer depths and oscillation frequencies, experimental data for frictional particles near the onset of patterns were found to be fit by the function $\lambda^{*}=1.0+1.1 f^{*-1.32 \pm 0.03}$, where $\lambda^{*}=\lambda / H$ 32].

We investigate the frequency dependence of standing waves in continuum simulations and in MD simulations of frictionless particles. Dimensionless accelerational amplitude $\Gamma=2.2$ was held constant while dimensionless frequency $f^{*}$ was varied. Simulations were conducted in a box of horizontal extent $L_{x}=168 \sigma$ and $L_{y}=10 \sigma$. This orientation causes stripe patterns to form parallel to the $y$ - axis. The dominant wavelength in each case was calculated from $S\left(k_{x}, k_{y}, t\right)$ by finding the wavenumber $k_{x}$ in the $x$ - direction which exhibited the maximum power during one cycle of the oscillatory state of the pattern. Due to the periodic boundary conditions and finite box size, wavelengths must fit in the box an integer number

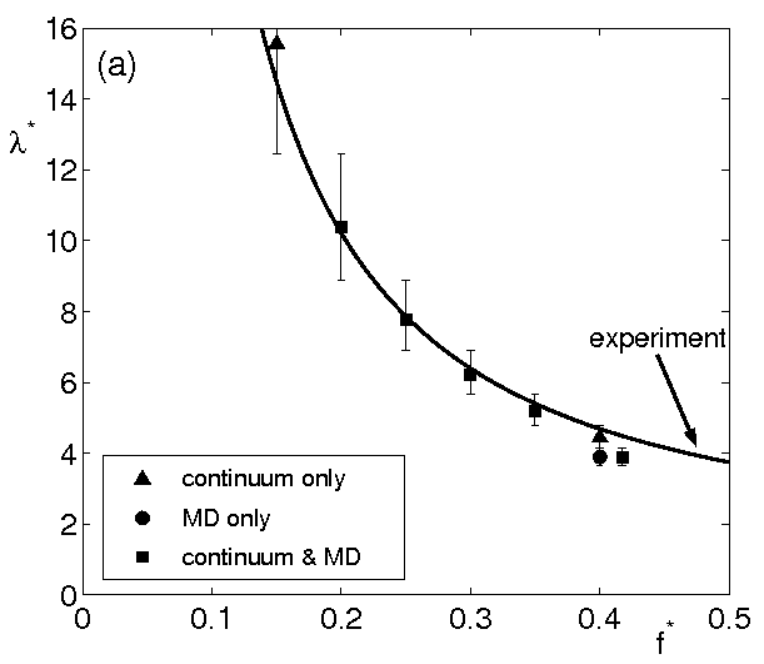

FIG. 2: Dispersion relation for stripes which form perpendicular to the long dimension of cells with horizontal dimensions $168 \sigma \times 10 \sigma$. Data for continuum simulations are shown as triangles and MD simulations as circles; points where continuum and MD simulations yield the same wavelength are shown as squares. In both continuum and MD simulations, the dominant wavelength of the final oscillatory state $\lambda$ fits very well to the dispersion relation found in experiments $\lambda^{*}=1.0+1.1 f^{-1.32 \pm 0.03}$ (solid line) 32]. Error bars in both simulations are calculated exclusively from discretization due to periodic boundary conditions in a finite size box.

of times. This finite size effect of quantized wavelength yields inherent uncertainty in the wavelength that would be selected in an infinite box.

Wavelengths found in continuum and MD simulations are compared to the dispersion relation fit to experimental data in Fig. 2. Investigation is limited to $f^{*}>0.15$ by the box size, as only two wavelengths fit in the box in continuum simulations at this frequency. Neither simulation produced patterns for this box size for $f^{*} \gtrsim 0.45$. Both simulations agree quite well with the experimental fit throughout the range $0.15 \lesssim f^{*} \lesssim 0.45$.

Comparison to the experimental fit shows that both $\mathrm{MD}$ and continnum simulations produce wavelengths consistent with experimental results for frictional particles. These data indicate that friction seems to be unimportant in wavelength selection through this parameter range.

\section{Layers Above and Below the Onset of Patterns}

Continuum and MD simulations exhibit pattern formation above a critical acceleration of the plate; however, standing wave patterns are not observed below a critical value of $\Gamma$ (Fig. 3). For $\Gamma=2.2$, both MD (Fig. 3(b)) and continuum (Fig. $3(\mathrm{~d})$ ) simulations show well defined peaks and valleys which form stripe patterns with two wavelengths fitting in the box of size $L_{x}=L_{y}=42 \sigma$. 


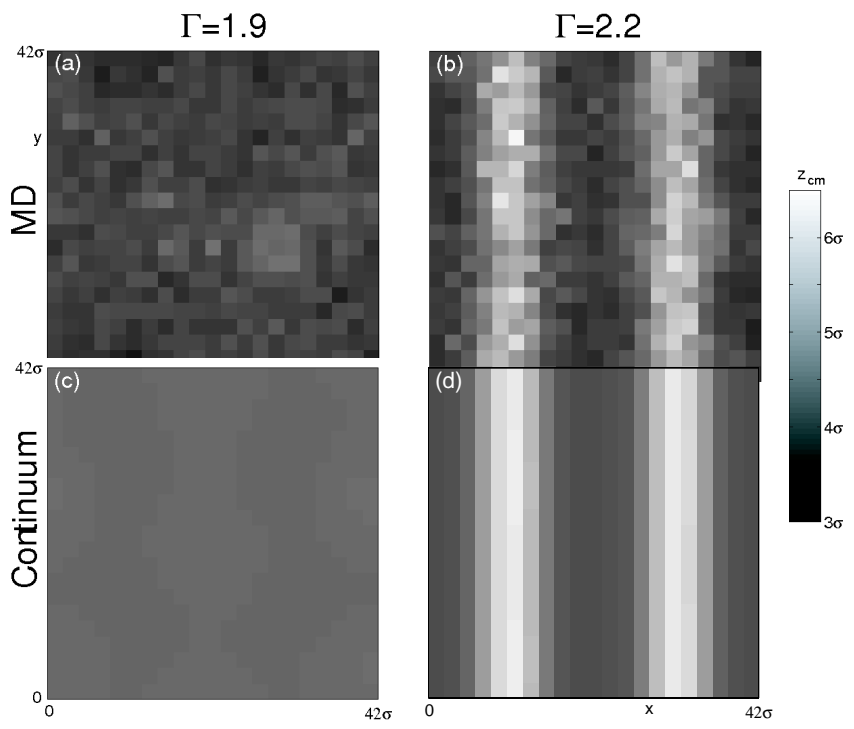

FIG. 3: An overhead view of the layer of grains, showing the center of mass height $z_{c m}(x, y)$ of the layer as a function of location in the box, for (a) MD simulations with a plate acceleration with respect to gravity $\Gamma=1.9$, (b) MD simulations with $\Gamma=2.2$, (c) continuum simulations with $\Gamma=1.9$, and (d) continuum simulations with $\Gamma=2.2$. Peaks corresponding to large $z_{c m}$ are shown in white, while valleys corresponding to small $z_{c m}$ are shown in black. The grayscale for all four images is given on the right.

The only difference between this system and that investigated in Sec. IIIA is the horizontal size of the cell; these patterns look very similar to a section of the patterns formed in the larger cell (Fig. 10. Reducing the accelerational amplitude to $\Gamma=1.9$ while keeping all other parameters constant yields no ordered waves in either MD (Fig. 3(a) or continuum (Fig. 3(c) . Thus both continuum and MD simulations appear to have a critical value of $\Gamma$ somewhere in the range $1.9 \leq \Gamma_{c} \leq 2.2$, such that no patterns are formed for $\Gamma<\Gamma_{c}$, and patterns are formed for $\Gamma>\Gamma_{c}$. This critical value is lower than that found in experiments with frictional particles, where a similar onset of patterns is found at a critical value of $\Gamma \approx 2.5$ [17.

Despite the similarities, differences between MD and continuum simulations are observable. For $\Gamma=1.9$, the continuum simulation yields a very smooth, flat layer (Fig. 3(c) ), while MD exhibits visible fluctuations (Fig. 3(a) . For $\Gamma=2.2$, the continuum simulations produce stripes (Fig. 3(d) which are much smoother than those found in MD simulation (Fig. 3(b)).

To explore the differences between the two simulations, we investigate the onset of patterns in more detail in continuum simulations and MD simulations separately.

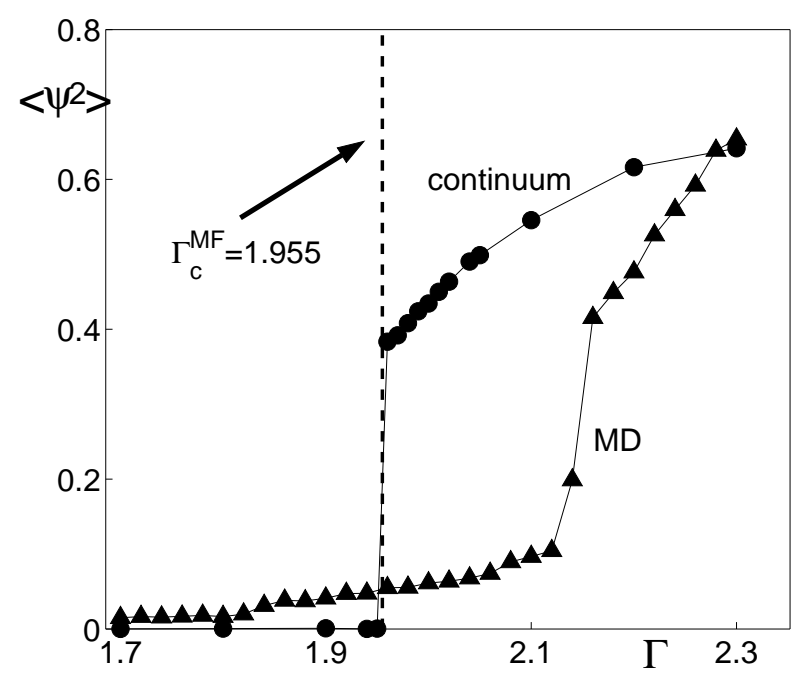

FIG. 4: The mean square deviation $\left\langle\psi^{2}\right\rangle$ of the local center of mass height from the average center of mass height of the entire layer as a function of accelerational amplitude $\Gamma$ for MD (triangles) and continuum (circles) simulations. The vertical dotted line represents the onset of stripe patterns in the continuum simulations.

\section{Onset of patterns in continuum simulations}

We investigate the onset of patterns in continuum simulations by determining $\left\langle\psi^{2}\right\rangle$ of standing waves for different values of $\Gamma$. Each simulation begins with a flat layer above the plate with small amplitude random fluctuations. The simulation is run until it reaches a periodic state, at which point $\left\langle\psi^{2}\right\rangle$ is calculated as an average over ten cycles of the same phase of the plate.

For $\Gamma \lesssim 1.95$, the initial fluctuations decay rapidly until the layer is quite flat, as represented by negligible values of $\left\langle\psi^{2}\right\rangle$ (Fig. (4). As $\Gamma$ increases, there is a sudden onset to large amplitude waves, as seen by the sudden jump in $\left\langle\psi^{2}\right\rangle$ in Fig. 4. This onset occurs at the critical value $\Gamma_{c}=1.955 \pm 0.005$. For $\Gamma<\Gamma_{c}$, initial fluctuations decay until the layer is very flat, while for all layers above onset $\left(\Gamma>\Gamma_{c}\right)$, these waves produce ordered patterns of stripes similar to those in Fig. 3(d)

\section{E. Onset of patterns in molecular dynamics simulations}

We examine the onset of patterns in MD simulations using the same methods as for the continuum equations. Figure 4 shows the mean square height deviation $\left\langle\psi^{2}\right\rangle$ as a function of $\Gamma$ for MD simulations as well as for continuum simulations. For each value of $\Gamma$, the simulation was run for 400 cycles of the plate until the layer reached a periodic state, then $\left\langle\psi^{2}\right\rangle$ and $P_{\max }$ were calculated from an average of the next 100 cycles.

As in continuum simulations, $\left\langle\psi^{2}\right\rangle$ grows with in- 
creasing $\Gamma$. Unlike the continuum results, $\left\langle\psi^{2}\right\rangle$ is nonnegligible in MD simulations even for $\Gamma<1.95$. There is still a sharp increase in the slope of the curve, but it is delayed until $\Gamma>2.1$.

\section{THE ROLE OF FLUCTUATIONS}

The MD simulations display an onset of ordered stripes that is delayed with respect to those found in continuum, and exhibit non-negligible $\left\langle\psi^{2}\right\rangle$ even below the onset of ordered stripes. Since the hydrodynamic model used in the continuum simulations does not include a stochastic noise term characteristic of fluctuating hydrodynamics, the differences between the continuum and MD simulations may be consistent with the presence of noise in the MD simulations due to the small number of particles per wavelength. To test the hypothesis that these differences are consistent with the presence of fluctuations in molecular dynamics simulations, we compare MD simulations to results from the Swift-Hohenberg model.

\section{A. Swift Hohenberg simulation}

The Swift-Hohenberg (SH) model was developed to describe thermal noise-driven phenomena near the onset of long range order in Rayleigh-Bénard convection [20]. Recent experimental evidence suggests similar phenomena in shaken granular experiments can be interpreted using the methods of fluctuating hydrodynamics [24].

The $\mathrm{SH}$ model describes the time evolution of a scalar field $\psi_{S H}(\mathbf{x}, \mathrm{t})$ :

$$
\frac{\partial \psi_{S H}}{\partial t}=\left(\epsilon-\left(1+\nabla^{2}\right)^{2}\right) \psi_{S H}-\psi_{S H}^{3}+\eta(\mathbf{x}, \mathrm{t}),
$$

where $\epsilon$ is the bifurcation parameter, and $\eta$ is a stochastic noise term of strength $F$, such that $\left\langle\eta(\mathbf{x}, \mathrm{t}) \eta\left(\mathbf{x}^{\prime}, \mathbf{t}^{\prime}\right)\right\rangle=$ $2 F \delta\left(\mathbf{x}-\mathbf{x}^{\prime}\right) \delta\left(t-t^{\prime}\right)$. In the absence of stochastic noise $(F=0)$, called the mean field (MF) approximation, there is a sharp onset of stripe patterns with long range order at $\epsilon=\epsilon_{c}^{M F}=0[20,33]$. For $F \neq 0$, the effect of noise is to delay the onset of long range (LR) order to a new critical value: $\epsilon_{c}^{L R}>0$. The delay in onset is characterized by $\Delta \epsilon_{c}=\epsilon_{c}^{L R}-\epsilon_{c}^{M F}$. In addition, the presence of noise creates fluctuations below the onset of long range order $\left(\epsilon<\epsilon_{c}^{L R}\right)$.

The Swift-Hohenberg simulation displays a forward bifurcation to stripes at onset, while MD simulations show slight $(<1 \%)$ hysteresis [24]. A more complicated SH model [34] yields square patterns with hysteresis; however, in this work we compare stripe formation in MD simulations a simpler model of the effects of noise near a bifurcation (Eq. 3).

We numerically solve the $\mathrm{SH}$ equation using the scheme described in [35], with the number of gridpoints $N=$ $42 \times 42$, and periodic boundary conditions. We use integration timesteps of 0.5 , and the size of each gridspace in the horizontal directions $\Delta x=\Delta y=0.29$ so that two wavelengths of the resulting pattern fit in the box, to match MD and continuum simulations. The simulation was allowed to run for 8,000 timesteps to reach a final pattern; then $\left\langle\psi_{S H}^{2}\right\rangle$ and $P_{\max }$ were calculated from an average of the next 2,000 timesteps, in the same way as $\left\langle\psi^{2}\right\rangle$ and $P_{\max }$ were calculated for MD and continuum simulations in Section IIC.

\section{B. Comparing Swift-Hohenberg and molecular dynamics simulations}

To find the strength of the noise and the mean field onset, we fit the $\mathrm{SH}$ model to the data from MD simulations (Fig. (5)) by varying three parameters: $F, \Delta \epsilon_{c}$, and an overall scale factor, as in [23, 24].

Of the three parameters, only the noise strength $F$ changes the overall shape of the curve. For a given $F$, the SH simulation is run for a range of $-0.2 \leq \epsilon \leq 0.2$; $\psi_{S H}$ and $P_{\max }$ are calculated from the steady state solution for each value of $\epsilon$ and compared to MD simulations. For consistency, $\left\langle\psi^{2}\right\rangle$ and $P_{\max }$ are calculated for MD simulations from bins of size $\Delta x_{b i n}=\Delta y_{b i n}=\sigma$ throughout this section, so that the number of bins in both MD and SH simulations is $42 \times 42$. Increasing the bin size to $\Delta x_{b i n}=\Delta y_{b i n}=2 \sigma$ does not change any of the fit parameters to within our uncertainty.

Note $\left\langle\psi_{S H}^{2}\right\rangle$ in $\mathrm{SH}$ simulations is found as a function of control parameter $-0.2 \leq \epsilon_{S H} \leq 0.2$, while in MD simulations, $\left\langle\psi_{M D}^{2}\right\rangle$ is found as a function of control parameter $1.7 \leq \Gamma \leq 2.3$. To compare the onset of the $\mathrm{SH}$ model to the onset in MD simulations, we define $\epsilon_{M D}=\left(\Gamma-\Gamma_{C}^{M F}\right) / \Gamma_{C}^{M F}$, where $\Gamma_{C}^{M F}$ is the mean field onset of patterns, comparable to $\epsilon_{S H}=0$. However, we do not know a priori the value of $\Gamma_{C}^{M F}$.

We find that $\left\langle\psi^{2}\right\rangle$ changes relatively smoothly in MD and $\mathrm{SH}$ simulations, making it difficult to pinpoint an onset of patterns from $\left\langle\psi^{2}\right\rangle$ alone. However, there is a distinct onset of long range order in the system (Fig. [5). For low $\Gamma$ in MD, the fluctuations are disordered (cf Fig. 3(a), while for higher $\Gamma$, standing wave stripe patterns are observed ( $c f$ Fig. 3(b)). A clear transition from disordered fluctuations to an ordered stripe pattern is demonstrated by the sharp increase in $P_{\max }$ as $\Gamma$ crosses the critical value for long range order, determined from Fig. 5(b) as $\Gamma_{c}^{L R}=2.15 \pm 0.01$. A similar transition to ordered stripes is seen in SH simulations (Fig. $5(\mathrm{~b})$ ).

The onset of long range order is used to establish a correspondence between $\Gamma$ and $\epsilon$. For MD simulations, we measure the onset of long range order as the point of sharpest increase in $P_{\max }$ (Fig. 5(b)]. In SH simulations, $\Delta \epsilon_{c}$ represents the onset of long range order. We match the single point of steepest increase of $P_{\max }$ between the two curves. The measured value $\Delta \epsilon_{c}$ in SH then predicts the mean field onset $\Gamma_{C}^{M F}$ corresponding to $\epsilon=0$.

Once the relationship between $\Gamma$ and $\epsilon$ is determined, the overall scale factor for a given $F$ is found by a least 

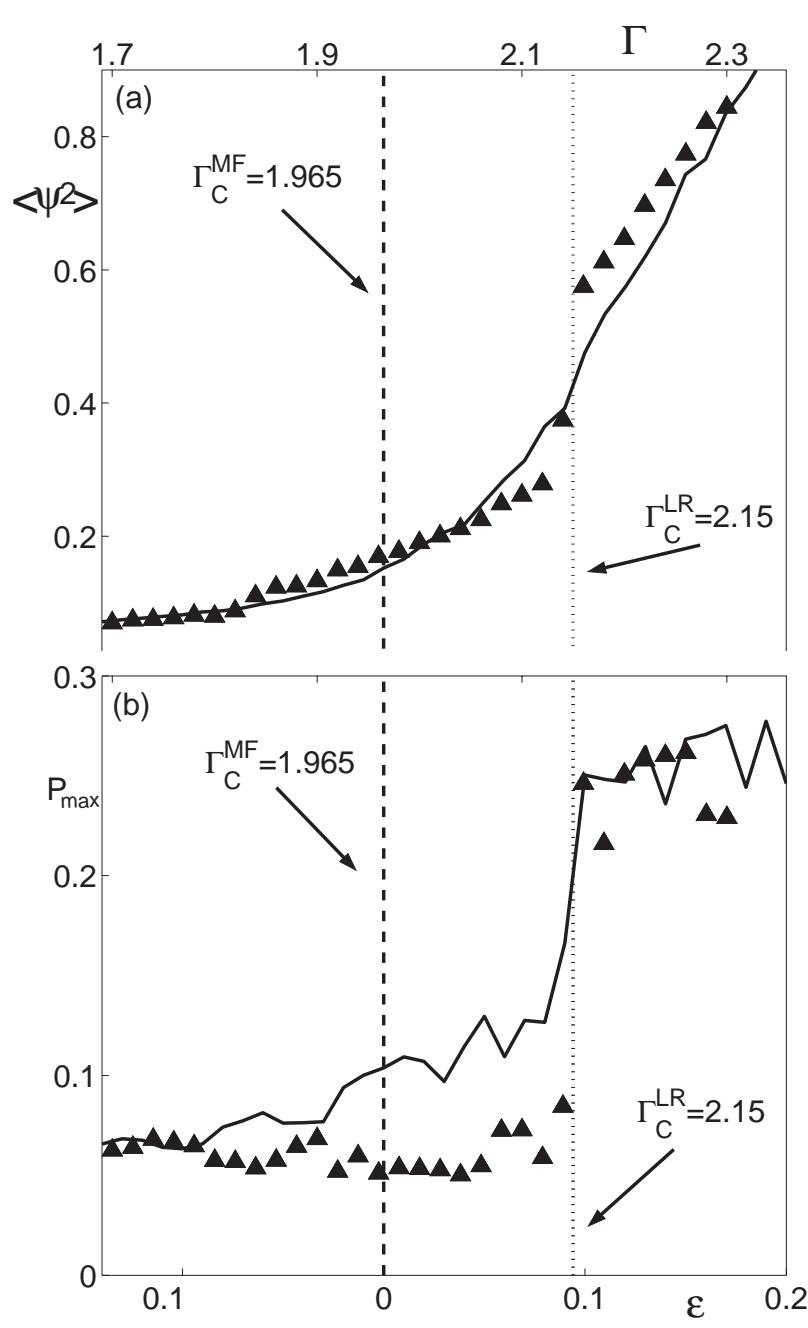

FIG. 5: Comparison of MD simulations (triangles) to the Swift-Hohenberg model (solid lines) for (a) $\left\langle\psi^{2}\right\rangle$, and (b) global ordering $P_{\max }$ (Eq. 2), as a function of control parameter $\epsilon$ (bottom axis) for $\mathrm{SH}$, and $\Gamma$ (top axis) for $\mathrm{MD}$. The parameters for $\mathrm{SH}$ simulations are noise strength $F=$ $(1.2 \pm 0.2) \times 10^{-2}$ and a delayed onset of long range or$\operatorname{der} \epsilon_{c}^{L R}=0.094$. The global ordering jumps sharply at $\epsilon_{c}^{L R}=0.094$, corresponding to $\Gamma_{c}^{L R}=2.15$ in $\mathrm{MD}$ (the vertical dotted line in the figure), representing a transition to stripe patterns, while $\left\langle\psi^{2}\right\rangle$ increases smoothly through that transition. This fit predicts a mean field onset value of $\Gamma_{c}^{M F}=1.965 \pm 0.007$, corresponding to $\epsilon_{c}^{M F}=0$ (the vertical dashed line in the figure).

squares fit between $\left\langle\psi_{S H}^{2}\right\rangle$ and $\left\langle\psi_{M D}^{2}\right\rangle$ for the range $1.7 \leq \Gamma \leq \Gamma_{c}^{L R}$ (see Fig. 5(b)). This minimization procedure gives the best possible fit for a given value of $F$.

This entire procedure is repeated for varying $F$, minimizing the squared residual $R^{2}=$ $\sum\left(\left\langle\psi_{M D}^{2}\right\rangle-\left\langle\psi_{S H}^{2}\right\rangle\right)^{2} / N$, where $N$ is the number of bins (Fig. 6). The best fit yields an onset of long range order at $\Delta \epsilon_{c}=0.94$, corresponding to $\Gamma_{c}^{L R}=2.15$. Figure 5(a) shows $\left\langle\psi^{2}\right\rangle$ as a function of $\epsilon$ for $\mathrm{SH}$

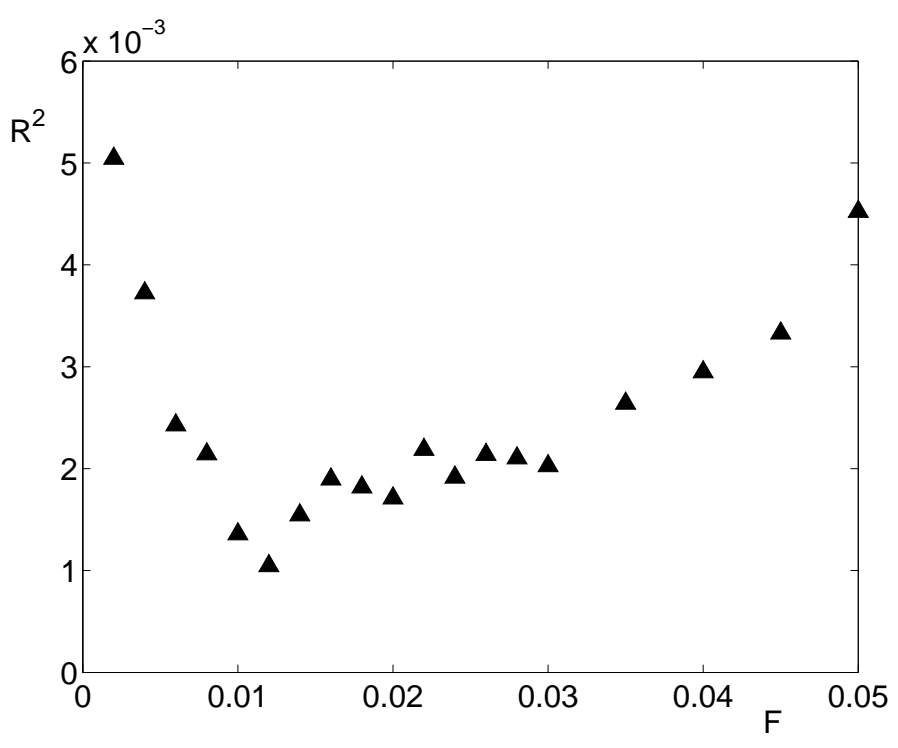

FIG. 6: The squared residual $R^{2}$ between $\left\langle\psi_{M D}^{2}\right\rangle$ and $\left\langle\psi_{S H}^{2}\right\rangle$ as a function of the noise strength $F$ used in $\mathrm{SH}$ simulations. The best least squares fit is given by $F=(1.2 \pm 0.2) \times 10^{-2}$.

simulations, and as a function of $\Gamma$ for MD simulations. The fit shows good agreement in $\left\langle\psi^{2}\right\rangle$ below $\epsilon=0$ (Fig. 5). Although the parameters are fit only in the range 1.7 $\leq \Gamma \leq \Gamma_{c}^{L R}$, agreement is reasonable even for $\Gamma>\Gamma_{c}^{L R}$.

The three parameter fit not only allows for agreement in $\left\langle\psi^{2}\right\rangle$, but also matches the measure of order $P_{\max }$ in the SH model to that found in MD simulation (Fig. 5(b)). In both MD and SH simulation, below the critical value of long range order, the fluctuations are disordered, leading to a small value in $P_{\max }$. When $\Gamma$ crosses the critical value, $P_{\max }$ jumps up significantly, and the observed patterns are ordered stripes. Below the onset of stripes, when the fluctuations are constantly shifting and changing, there is significant uncertainty in finding the value of $P_{\max }$, as seen by the noisy curve on the plot. Above this onset, however, the standing waves produce stable stripes, and $P_{\max }$ plateaus and remains quite constant, with good agreement between MD and SH simulations. Finally, the mean field onset $\Gamma_{c}^{M F}=1.965 \pm 0.007$ predicted by this fit agrees remarkably well with the critical value $\Gamma_{c}=1.955 \pm 0.005$ found in our simulations of Navier-Stokes order continuum equations.

\section{Effect of changing wavelength on strength of noise}

If the noise effects arise from the finite particle number in $\mathrm{MD}$, we may expect that this effect will decrease in systems in which there are more particles per wavelength of pattern. Since the number of particles in a volume $\lambda^{3}$ increases with increasing wavelength, we investigate the 


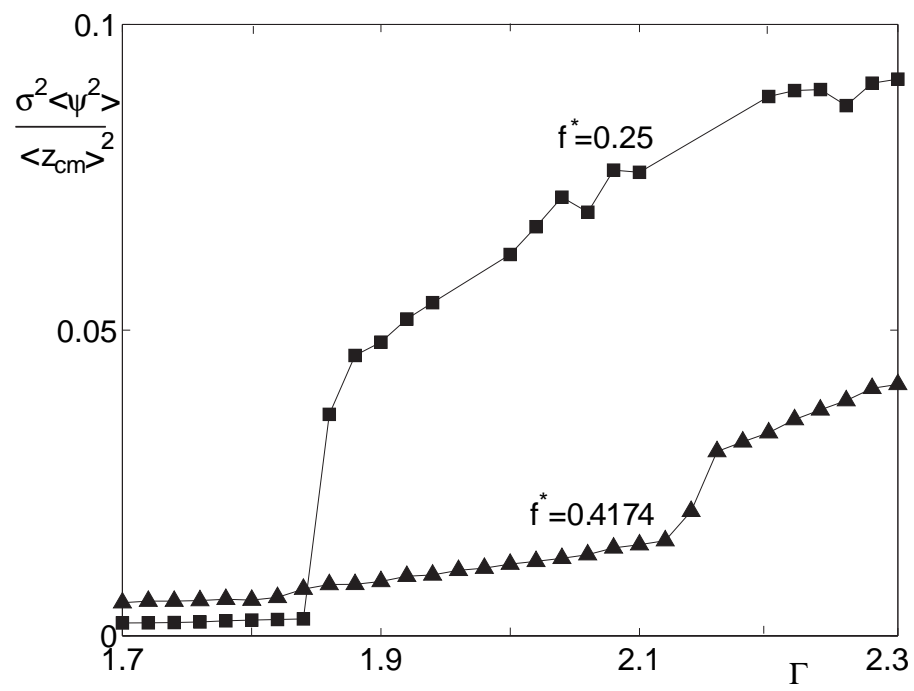

FIG. 7: Comparison growth of $\left\langle\psi_{S H}^{2}\right\rangle$ normalized by the mean center of mass height of the layer $\sigma^{2}\left\langle\psi^{2}\right\rangle /\left\langle z_{c m}\right\rangle^{2}=$ $\left\langle\left(z_{c m}-\left\langle z_{c m}\right\rangle\right)^{2}\right\rangle /\left\langle z_{c m}\right\rangle^{2}$ for MD simulations with $f^{*}=0.25$ (squares) and $f^{*}=0.4174$ (triangles). The lower frequency displays much smaller fluctuations below the onset of patterns than does the higher frequency.

effect of changing frequency on the onset of patterns in MD simulations. For cells of horizontal extent $168 \sigma \times 10 \sigma$, layers shaken with a frequency $f^{*}=0.25$ form peaks with a dominant wavelength $\lambda=42 \sigma$, which is twice the wavelength found for patterns investigated at $f^{*}=$ 0.4174 (see Fig. 2).

We examine layers shaken at $f^{*}=0.25$ in cells of size $L_{x}=L_{y}=2 \lambda=84 \sigma$, while holding constant layer depth $H=5.4$ and restitution coefficient $e=0.70$. We vary $\Gamma$ through the same range $1.7 \leq \Gamma \leq 2.3$ investigated for $f^{*}=0.4174$ earlier in this paper. Figure 7 shows the growth of $\left\langle\psi_{S H}^{2}\right\rangle$ normalized by the mean center of mass height of the layer squared $\sigma^{2}\left\langle\psi^{2}\right\rangle /\left\langle z_{c m}\right\rangle^{2}=$ $\left\langle\left(z_{c m}-\left\langle z_{c m}\right\rangle\right)^{2}\right\rangle /\left\langle z_{c m}\right\rangle^{2}$ for MD simulations with frequencies $f^{*}=0.25$ and $f^{*}=0.4174$.

The lower frequency $\left(f^{*}=0.25\right)$ exhibits a much sharper jump in $\left\langle\psi_{S H}^{2}\right\rangle$ than that seen at $f^{*}=0.4174$. Below this onset, the curve is much flatter for $f^{*}=0.25$, while at $f^{*}=0.4174$, the curve increases much more gradually through onset. Proportionally smaller fluctuations compared to pattern amplitude is consistent with lower noise strength for $f^{*}=0.25$ than that found for $f^{*}=0.4174$. In addition, the rapid growth of peaks and valleys occurs at a smaller value of $\Gamma$ for $f^{*}=0.25$, corresponding to an onset even below the mean field onset $\Gamma_{c}^{M F}$ for the larger frequency.

We follow the same procedure as for $f^{*}=0.4174$ to fit the data from MD simulation to the Swift-Hohenberg model. We note that for frictional particles, square patterns are formed for $f^{*}=0.25$; in the absence of fric-
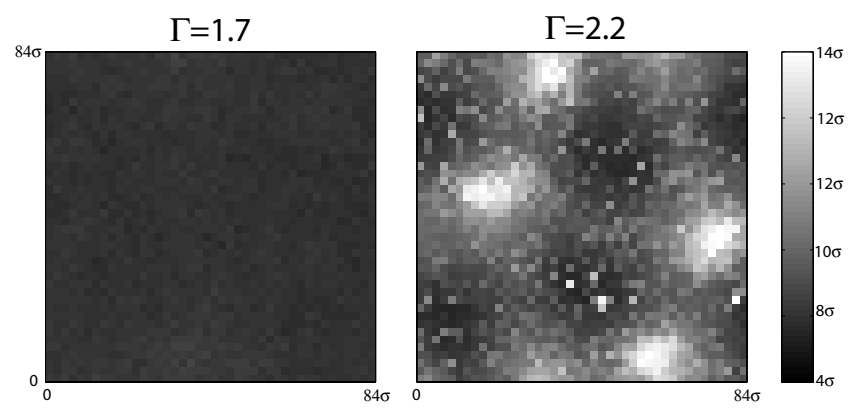

FIG. 8: An overhead view of the layer of grains from MD simulations at $f^{*}=0.25$, for $\Gamma=1.7$ and $\Gamma=2.2$. Note how much less noise there is below onset here $(\Gamma=1.7)$ compared to the results for $f^{*}=0.4174$ in Fig. 3 The images show the center of mass height $z_{c m}(x, y)$ of the layer as a function of location in the box. These MD simulations use a cell which is $L_{x}=L_{y}=84 \sigma$ in the horizontal directions. Peaks corresponding to large $z_{c m}$ are shown in white, while valleys corresponding to small $z_{c m}$ are shown in black. The grayscale for both images is given on the right.

tion, peaks and valleys remain disordered, and no regular square lattice forms in experiments or MD simulations [25, 26] (see Fig. 8). Thus the onset of long range order is ill defined in this case. However, this lower frequency exhibits a much sharper onset in the growth of $\left\langle\psi_{S H}^{2}\right\rangle$, which is used to find $\Delta \epsilon_{c}$. The best fit yields a noise term $F=(4 \pm 3) \times 10^{-4}$, and a mean field onset of $\Gamma_{c}^{M F}=1.85 \pm 0.01$. Our hydrodynamic simulations find the flat layer becomes unstable at $\Gamma_{c}=1.84 \pm 0.01$, which again agrees well with the mean field onset found from the fit to the SH model.

The noise strength at $f^{*}=0.4174$ is approximately 30 times larger than the noise strength at $f^{*}=0.25$. This leads to qualitatively different behavior of $\left\langle\psi_{S H}^{2}\right\rangle$ near onset, yielding a smoother curve for the higher frequency and a sharper onset for lower frequency. Finally, the onset is barely delayed for the lower frequency, with $\Delta \epsilon_{c}=0.01$ for $f^{*}=0.25$, as compared to $\Delta \epsilon_{c}=0.10$ for $f^{*}=0.4174$.

Thus a change in frequency which increases the wavelength at onset by a factor of 2 decreases the amount of noise by a factor of 30. For Rayleigh-Bénard convection in ordinary fluids, the functional dependence of $F$ on $n$, $\mathbf{u}, T$, and $\lambda$ is known [36, 37]. However, this dependence is not known for oscillated granular layers. Future investigation of the dependence of $F$ on shaking parameters $f^{*}, \Gamma$, and $H$, or on hydrodynamic variables $n, \mathbf{u}, T$ in experiment and MD simulations may provide information on the dependence of the noise strength $F$ that can be used in continuum simulations.

\section{CONCLUSIONS}

We have shown that continuum simulations can describe important aspects of pattern formation in granular 
materials. For a nondimensional frequency $f^{*}=0.4174$, both MD and continuum simulations of granular materials form stripe patterns of the same wavelength above a critical value $\Gamma>\Gamma_{c}$, and display no stripes for $\Gamma<\Gamma_{c}$. Further, the two simulations yield the same dependence of wavelength on frequency. These wavelengths agree with the dispersion relation found experimentally for frictional particles.

The effect of fluctuations has been examined in simulations of the Swift-Hohenberg model. The results deduced for the mean field onset in MD simulations agree well with the actual onset in continuum simulations for both $f^{*}=0.4174$ and $f^{*}=0.25$.

We find the strength of the noise to be $F=$ $(1.2 \pm 0.2) \times 10^{-2}$ for stripes at $f^{*}=0.4174$, and $F=$ $(4 \pm 3) \times 10^{-4}$ for disordered squares at $f^{*}=0.25$. The value determined in an experiment for a slightly shallower granular layer at $f^{*}=0.28$ was $F=3.5 \times 10^{-3}$ [24], which is within the range of noise values obtained in this investigation. The smallest noise strength found for our granular system is comparable to the largest noise strength found thus far in experiments in ordinary fluids, which obtained $F=7.1 \times 10^{-4}$ in measurements near the critical point, while values typical for convection are closer to $10^{-9}$ [23]. For $f^{*}=0.4174$, the noise is strong enough to delay onset of long range patterns by $10 \%$ in MD simulation, and influences strongly the behavior of the system even more than $20 \%$ below this onset. Thus noise plays an important role in granular media near the onset of patterns.
This study indicates that hydrodynamic theory holds promise for investigating and understanding pattern formation in granular flows. However, quantitative comparisons between continuum theory and experiment will require the addition of noise terms into the equations. The addition of noise would be an important step towards using the powerful tools of hydrodynamic theory to investigate problems of pattern formation in granular materials.

The absence of friction in these simulations restricts our investigation to stripe patterns. Simulations without friction have not yielded the square and hexagonal patterns seen in experiments with frictional particles [26]. Further research into pattern formation using continuum simulations should investigate the most effective way to incorporate friction between particles into the continuum simulations and should examine how the strength of friction in the simulation affects pattern formation in the system.

\section{Acknowledgments}

We thank Daniel I. Goldman, W. D. McCormick, Sung Joon Moon, and Erin C. Rericha for helpful discussions. This work was supported by the Engineering Research Program of the Office of Basic Energy Sciences of the Department of Energy (Grant DE-FG03-93ER14312).
[1] L. Bocquet, W. Losert, D. Schalk, T. C. Lubensky, and J. P. Gollub, Phys. Rev. E 65, 011307 (2001).

[2] E. C. Rericha, C. Bizon, M. D. Shattuck, and H. L. Swinney, Phys. Rev. Lett. 88, 014302 (2002).

[3] R. Ramírez, D. Risso, R. Soto, and P. Cordero, Phys. Rev. E 62, 2521 (2000).

[4] J. W. Dufty, in Challenges in Granular Physics, edited by T. Halsey and A. Mehta (World Scientific (also available at cond-mat/0108444), 2002).

[5] C. S. Campbell, Annu. Rev. Fluid Mech. 22, 57 (1990).

[6] H. L. Swinney and E. C. Rericha, in The Physics of Complex Systems (New Advances and Perspectives)- The International School of Physics Enrico Fermi, edited by F. Mallamace and E. Stanley (IOS Press, Amsterdam (also available at cond-mat/0408252), 2004).

[7] P. K. Haff, J. Fluid Mech. 134, 401 (1983).

[8] J. T. Jenkins and S. B. Savage, J. Fluid Mech. 130, 187 (1983).

[9] C. K. K. Lun, S. B. Savage, D. J. Jeffrey, and N. Chepurniy, J. Fluid Mech. 140, 233 (1984).

[10] A. Goldshtein and M. Shapiro, J. Fluid Mech. 282, 75 (1995).

[11] J. T. Jenkins and M. W. Richman, Arch. Rat. Mech. Anal. 87, 355 (1985).

[12] N. Sela and I. Goldhirsch, J. Fluid Mech. 361, 41 (1998).

[13] J. B. Knight, E. E. Ehrichs, V. Y. Kuperman, J. K. Flint, H. M. Jaeger, and S. R. Nagel, Phys. Rev. E 54, 5726
(1996).

[14] E. Falcon, R. Wunenburger, P. Évesque, S. Fauve, C. Chabot, Y. Garrabos, and D. Beysens, Phys. Rev. Lett. 83, 440 (1999).

[15] A. Goldshtein, M. Shapiro, L. Moldavsky, and M. Fichman, J. Fluid Mech. 287, 349 (1995).

[16] J. J. Brey, M. J. Ruiz-Montero, and F. Moreno, Phys. Rev. E 63, 061305 (2001).

[17] F. Melo, P. Umbanhowar, and H. L. Swinney, Phys. Rev. Lett. 72, 172 (1994).

[18] L. D. Landau and E. M. Lifshitz, Fluid Mechanics (Pergamon Books Ltd., Oxford, 1959).

[19] V. M. Zaitsev and M. I. Shilomis, Soviet Physics JETP 32, 866 (1971).

[20] J. B. Swift and P. C. Hohenberg, Phys. Rev. A 15, 319 (1977).

[21] M. Wu, G. Ahlers, and D. S. Cannell, Phys. Rev. Lett. 75, 1743 (1995).

[22] I. Rehberg, S. Rasenat, M. de la Torre Juárez, W. Schöpf, F. Hörner, G. Ahlers, and H. R. Brand, Phys. Rev. Lett. 67, 596 (1991).

[23] J. Oh and G. Ahlers, Phys. Rev. Lett. 91, 094501 (2003).

[24] D. I. Goldman, J. B. Swift, and H. L. Swinney, Phys. Rev. Lett. 92, 174302 (2004).

[25] D. I. Goldman, M. D. Shattuck, S. J. Moon, J. B. Swift, and H. L. Swinney, Phys. Rev. Lett. 90, 104302 (2003).

[26] S. J. Moon, J. B. Swift, and H. L. Swinney, Phys. Rev. 
E 69, 031301 (2004).

[27] C. Bizon, M. D. Shattuck, J. B. Swift, W. D. McCormick, and H. L. Swinney, Phys. Rev. Lett. 80, 57 (1998).

[28] J. Bougie, S. J. Moon, J. B. Swift, and H. L. Swinney, Phys. Rev. E 66, 051301 (2002).

[29] S. J. Moon, M. D. Shattuck, C. Bizon, D. I. Goldman, J. B. Swift, and H. L. Swinney, Phys. Rev. E 65, 011301 (2002).

[30] F. Melo and S. Douady, Phys. Rev. Lett. 71, 3283 (1993).

[31] E. Clément, L. Vanel, J. Rajchenbach, and J. Duran, Phys. Rev. E 53, 2972 (1996).

[32] P. Umbanhowar and H. L. Swinney, Physica A 288, 344
(2000)

[33] M. A. Scherer, G. Ahlers, F. Hörner, and I. Rehberg, Phys. Rev. Lett. 85, 3754 (2000).

[34] H. Sakaguchi and H. R. Brand, Europhys. Lett. 38, 341 (1997).

[35] M. C. Cross, D. Meiron, and Y. Tu, Chaos 4, 607 (1994).

[36] P. C. Hohenberg and J. B. Swift, Phys. Rev. A 46, 4773 (1992).

[37] H. van Beijeren and E. G. D. Cohen, J. Stat. Phys. 53, 77 (1988) 